\title{
Gamma-ray binaries: binary systems dominated by their gamma-ray emission
}

\author{
Brian van Soelen* \\ University of the Free State \\ E-mail: vansoelenbeufs.ac.za
}

\begin{abstract}
Gamma-ray binaries are a new class of binary systems, discovered over the last few decades due to the progress made in gamma-ray astronomy. These sources are characterised by spectral energy distributions that are dominated by emission at gamma-ray energies, distinguishing them from other gamma-ray detected binaries. There are currently only six known systems, with a few candidate sources identified. The model most often used to explain these sources is that they consist of a non-accreting pulsar, and that the non-thermal emission originates from the shock which forms between the pulsar and stellar winds. However, a number of questions still remain regarding the exact location and production mechanism behind the emission in these binaries. Here I present a review of gamma-ray binaries, highlighting some recent achievements made, as well as some challenges that still need to be addressed, particular in the era of CTA.
\end{abstract}

5th Annual Conference on High Energy Astrophysics in Southern Africa

4-6 October, 2017

University of the Witwatersrand (Wits), South Africa

${ }^{*}$ Speaker. 


\section{Introduction}

Gamma-ray binaries are a distinct class of high-mass binary systems that have spectral energy distributions (SEDs) that peak in the gamma-ray regime (in a $v F_{v}$ representation). This distinguishes them from other binary systems that have been detected in gamma rays, but where the output peaks at lower energies. This is a small class of sources and there are currently only six well established systems. All of these systems consist of a compact object, in the mass range of a neutron star or black hole, in orbit around an O or B type star. For only one source, PSR B125963/LS 2883, is the nature of the compact object confirmed to be a pulsar. Two candidate systems have been proposed, and for one of these, PSR J2032+4127/MT91 213, increased gamma-ray emission around periastron has been reported by the MAGIC and VERITAS gamma-ray telescopes $[1,2]$. If this is confirmed, it will be the second gamma-ray binary where the nature of the compact object is known. For all the other systems no pulsed emission has been detected, the detection of which would confirm the compact object is a neutron star. The most recently discovered gammaray binary, LMC P3, is located in the large Magellanic cloud (LMC), the most luminous of these sources, and the first discovered outside of the Galaxy [3]. Initially discovered from a period search in Fermi-LAT observations, the source has recently been detected by H.E.S.S., though only at one phase bin [4].

The orbital parameters of the six gamma-ray binaries, as well as the best candidate PSR J2032+4127/MT91 213, are summarized in Table 1.

\section{Modelling gamma-ray binaries}

All gamma-ray binaries show very similar SEDs, with non-thermal emission detected for most sources from radio up to TeV energy gamma-rays, with all binaries having been detected at both high energy $(0.1-100 \mathrm{GeV})$ and very high energy ( $>100 \mathrm{GeV}$; VHE) gamma rays. The origin of the non-thermal emission depends on the nature of the compact object. If the compact object is a black hole, the emission would be accretion driven, and these would be microquasar-like systems. Gamma rays have been detected from microquasars, i.e. Cygnus X-1 and Cygnus X-3, but the SEDs are different to those of gamma-ray binaries, since they peak at X-ray energies (e.g. [20, 21]).

Alternatively, if the compact objects are pulsars, like in the case of PSR B1259-63/LS 2883, then the emission is powered by the spin-down of the pulsar and the interaction between the pulsar and stellar wind in the binary. PSR B1259-63 was first modelled this way by Tavani et al. [22] and the scenario is similar to that of pulsar wind nebulae (e.g. [23]). In this model there is no accretion onto the pulsar and a shock forms between the stellar and pulsar winds, with the pulsar wind "wrapped" around the pulsar. The subsequent particle acceleration in the shock produces the non-thermal emission.

For PSR B1259-63 this pulsar wind scenario is known to be powering the emission and the similarities between the different gamma-ray binaries points to the same mechanisms occurring in all the systems. Dubus [24] put forward this argument and showed how the emission from all of the, then known, systems could be modelled in this way (Fig. 1). The author also argues that none of the sources showed the variability expected from accretion driven systems and do not show a cutoff in the X-ray part of the regime typical of accretion driven systems. Further, while pulsed radio 
Table 1: The orbital parameters of the gamma-ray binaries.

\begin{tabular}{lcccc}
\hline & Orbital Period (d) & $e$ & Optical companion & references \\
\hline PSR B1259-63 & $1236.724526(6)$ & $0.86987970(6)$ & O9.5Ve & {$[5,6]$} \\
LS 5039 & $3.90603 \pm 0.00017$ & $0.35 \pm 0.04$ & O6.5V & {$[7]$} \\
LS I +61 303 & $26.4960 \pm 0.0028$ & $0.537 \pm .034$ & B0Ve & {$[8,9,10,11]$} \\
HESS J0632+057 & $315_{-4}^{+6}$ & $0.83 \pm 0.08$ & B0Ve & {$[12,13]$} \\
1FGL J1018.6-5856 & $16.544 \pm 0.008$ & $0.31 \pm 0.16$ & O6V $((\mathrm{f}))$ & {$[14,15,16]$} \\
LMC P3 & $10.301 \pm 0.002$ & $<0.7$ & O5III & {$[3,17]$} \\
PSR J2032+4127 & $45-50$ years & $>0.94$ & B0Ve & {$[18,19]$} \\
\hline
\end{tabular}
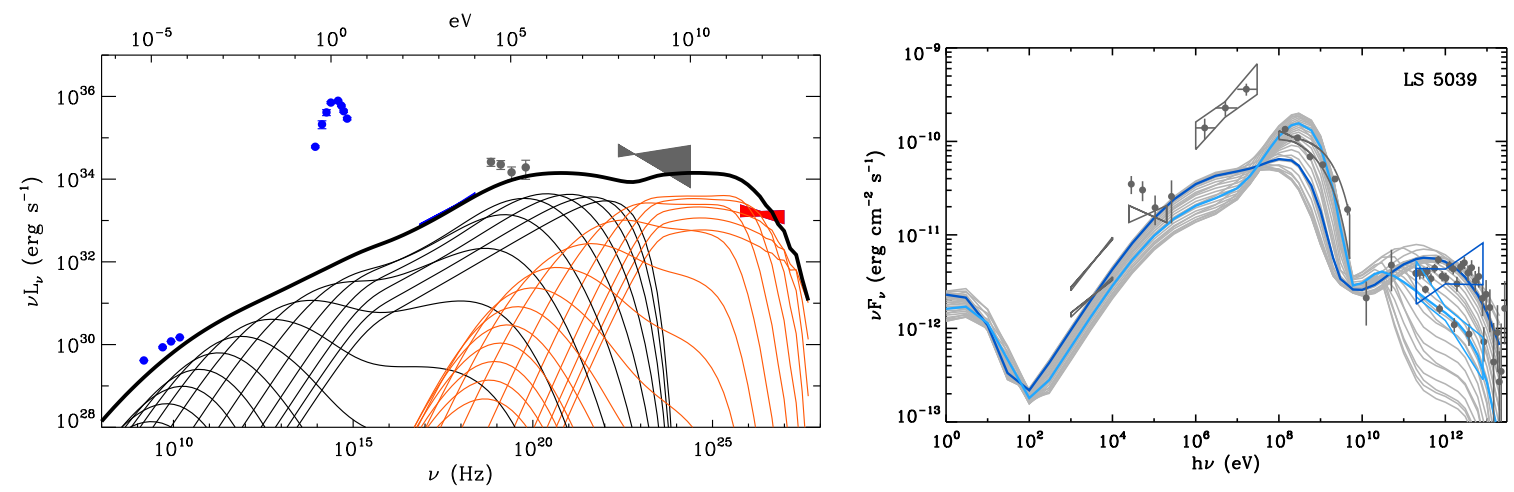

Figure 1: Left: Broad-band model of LS 5039 presented by Dubus [24]. The thin lines correspond to emission from different regions in the shocked wind, with the broad line showing the total emission (Credit: Dubus et al., A\&A, 456, 801, 2006, reproduced with permission (c) ESO) Right: More recent modelling of LS 5039 based on numerical relativistic hydrodynamic simulations by Dubus et al. [64]. (Credit: Dubus et al., A\&A, 581, A27, 2015, reproduced with permission (C) ESO)

signals have only been detected from PSR B1259-63, all the other systems have shorter orbital periods (Tab. 1) and the compact object is close enough to the optical companion that any pulsed radio signal would be quenched by free-free absorption in the stellar wind. This indeed happens for PSR B1259-63 when the pulsar passes close to the optical companion near periastron and there is an eclipse of the pulsed radio emissions [25].

The extended radio emission observed from gamma-ray binaries can be explained as emission from the extended shock, and not necessarily from a microquasar-like jet [24, 26]. However, it should be noted that for LS I $+61^{\circ} 303$ it has been argued that observations with the VLBA/VLBI show a precessing jet outflow, e.g. [27, 28, 29]. LS I $+61^{\circ} 303$ has also been observed to show strong orbit to orbit variability at VHE [30]. If all gamma-ray binaries are powered by the same pulsar wind mechanism, then the observed differences between the sources should be due to the different orbital parameters and orientations of the binaries (relative to the observer).

In this proceedings I will briefly highlight some of the challenges and topics that may be important as we move towards the era of gamma-ray astronomy with the Cherenkov Telescope Array (CTA) [31]. 


\section{Some challenges and opportunities}

Below some of the challenges, open questions and recent results are summarized.

\subsection{Constraining the orbital parameters}

One of the keys to modelling gamma-ray binaries is a knowledge of the orbital parameters. In the case of PSR B1259-63, the very long term monitoring of the pulsar allows highly accurate orbital periods to be determined [5]. However, in the other systems this is not possible. While the orbital period can often best be determined by period analyses of the multi-wavelength light curves (e.g. $[8,12,14,3])$ the other parameters (i.e. the eccentricity, longitude of periastron and mass function) are determined from the optically determined radial velocity of the more massive optical companion. This requires high resolution optical spectroscopy spread out with sufficient cadence over the orbital period of the source, with ground based telescopes that must operate in pointed mode.

The lack of these parameters can put constraints on attempting to model these sources. For example two recent models for 1FGL J1018.6-5856 [32, 33] both placed different constraints on the binary geometry, but both are slightly at odds with the subsequently reported velocity solution [15]. And while modelling combined with multi-wavelength observations can be used to place constraints on the orbital geometry, the most accurate results are still dependent on spectroscopically determined radial velocities. As another example the TeV source, HESS J1832-093, has been proposed to be a gamma-ray binary candidate, due to the discovery of a variable X-ray source co-incident with an infrared source, 2MASS J18324516-0921545 [34]. However, so far no binary period has been detected from the X-ray observations [35]. Spectroscopic observations are required to determine whether the infrared source presents periodic radial velocity variations, but these are hampered because of the high extinction towards the source. Any future detection of similar high extinction sources which lie within the Galactic plane would be hampered by the same problem and observations may be limited to mid-infrared (or longer) wavelengths.

\subsection{Explaining the $\mathrm{GeV}$ emission}

There is still some debate around the production of gamma-ray emission in the GeV energy range in gamma-ray binaries. Understanding the $\mathrm{GeV}$ emission is critical to understanding the physics in these binaries. In general, gamma-ray binaries show a correlation between the X-ray and TeV light curves (e.g. LS 5039 and LMC P3) but not with the GeV light curves [36, 37, 3, 4]. For example, in PSR B1259-63, while the X-ray and TeV light curves peak slightly before and after periastron, the $\mathrm{GeV}$ light curve shows little or no emission around periastron, but a post-periastron flare with a luminosity approaching the spin-down luminosity of the source [38, 39].

The GeV emission has been suggested to arise from the pulsar, since the SED is similar to that observed for gamma-ray pulsars (see e.g. the discussion in [40]), but searches have so far not detected any pulsations in the emission (e.g. [36, 38]). It has also been suggested that the $\mathrm{GeV}$ emission could arise from inverse Compton scattering of electrons in the "cold" pulsar wind (i.e. before the pulsar wind reaches the shock). Such electrons would be more mono-energetic than those re-accelerated in the shock and would, therefore, have a sharper peak in the GeV regime. Such models have been suggested to explain the flare observed in PSR B1259-63, where the seed 
photons originate from the shocked circumstellar disc [41] or from the synchrotron produced X-ray photons [42]. More recent models have also invoked a temporary accretion disc around the compact object in PSR B1259-63 and HESS J0632+057 as a source of seed photons (see section 3.4).

While the models for the scattering of the "cold" pulsar wind could explain the shape and apparent cut-off of the GeV emission in the SED of the source, an alternative explanation is that the particle acceleration is very efficient and electrons are able to accelerate up to energies constrained by the self-regulated cut-off, and the $\mathrm{GeV}$ emission is due to synchrotron radiation [43].

\subsection{The effect of Doppler boosting}

Bogovalov et al. [44] undertook relativistic hydrodynamic simulations of the interaction between the non-relativistic stellar wind and relativistic wind from the pulsar (for PSR B1259-63) and showed that the bulk motion of the outflow becomes mildly relativistic near the apex of the shock, but increases up to Lorentz factors of $\Gamma \sim 100$, moving away from the apex. This could make Doppler boosting an important contributing factor, depending on where the emission originates from in the binaries.

The effect of the Doppler boosting has subsequently been looked at by a number of authors. For example, Dubus et al. [45] looked at its influence in LS 5039, LS I +61 303 and PSR B125963, concluding that the Doppler boosting was important and may explain the X-ray light-curve of LS 5039. The effect of Doppler boosting was also considered by Kong et al. [46] in a model to explain the GeV flare observed in PSR B1259-63, since it occurs close to inferior conjunction. More recently, An \& Romani [32] and Chen et al. [33] presented models for 1FGL J1018.6-5856 proposing that the "spike" seen in the X-ray light curves must occur close to inferior conjunction and is due to Doppler boosting of the emission.

\subsection{Influence of the circumstellar disc}

In three of the gamma-ray binaries, the optical companion is a Be type star. Be stars are surrounded by circumstellar decretion discs (see e.g. Rivinius et al. [47] for a review of classical Be stars). In PSR B1259-63 the location of the circumstellar disc in the binary has been constrained by the on-set of the eclipse of the pulsed radio signal for $\sim 30$ days around periastron $[25,48,49,50]$, and corresponds to periods of maxima in the X-ray light curve [43]. The disc is also seen by the strong $\mathrm{H} \alpha$ emission line, which shows variation around periastron [50, 51]. In LS I +61 303 long term variation in the circumstellar disc is observed as a super-orbital variation in the $\mathrm{H} \alpha$ emission, with the same period as that detected in the non-thermal emission (e.g. [52]).

The presence of the circumstellar disc introduces a number of possibilities for influencing the production of non-thermal emission in gamma-ray binaries, a few of which will be briefly discussed below.

\subsubsection{The infrared excess}

The additional emission from the circumstellar disc results in the spectra of Be stars showing an "infrared excess" [47]. This excess provides additional soft photons which will influence the gamma-ray production. For example, these infrared photons provide an additional source of target photons for inverse Compton scattering, and since the target photons lie at lower energies, 
would produce additional gamma-rays in the $\mathrm{GeV}$ regime. However, modelling the resulting inverse Compton emission, using a model for the infrared flux constrained by mid-infrared observations near periastron, shows that while there is an increase, the emission peaks close to periastron and not near the disc crossings or the period of the Fermi flare $[53,54]$. Target photons originating from the disc were also used in the model of Khangulyan et al. [41] but would require a very high luminosity generated by possible shocks in the disc.

The infrared photons are also important for gamma-gamma absorption in the system as discussed in section 3.5

\subsubsection{Structure of the disc and shock}

The variability of the circumstellar disc has been predicted by smoothed particle hydrodynamic (SPH) simulations [55]. The changing structure will influence the size of the shock front that forms around the pulsar and should subsequently modify the emission. Since the position of the stand-off shock is determined by the strength of the stellar winds, the shock will change as the pulsar passes from the polar wind into the disc, introducing discontinuities in the light curves. This has been looked at in models such as that presented by Kong et al. [56] and in SPH modelling by Takata et al. [57].

The interaction between the pulsar wind and the circumstellar disc may also be directly linked to the GeV flare seen in PSR B1259-63. Optical spectroscopy showed that the $\mathrm{H} \alpha$ emission line (originating from the disc) changed rapidly at the on-set of the 2014 flare, which points to an interaction between these two components [43].

\subsubsection{Temporary accretion discs?}

Very recently Yi \& Cheng $[58,59]$ have proposed a new model to explaining the $\mathrm{GeV}$ emission in PSR B1259-63 and HESS J0632+057. Their model assumes that both systems contain a nonaccreting pulsar which pass through the Be stars' circumstellar discs twice per orbit (as is seen for PSR B1259-63). The model proposes that during this time, part of the circumstellar disc could be captured in the gravitational well of the pulsar and this material will start to form an accretion disc. As the disc forms, the inner edge will move inwards until the inner radius is close enough that the propeller effect will start to throw material out, decreasing the mass of the disc. While no accretion onto the pulsar occurs, a temporary accretion disc/ring will form. The disc will heat up, creating a source of seed photons for inverse Compton scattering from the cold pulsar wind. In the case of HESS J0632+057 their model requires a large circumstellar disc spread over a large part of the orbit.

\subsection{Gamma-gamma absorption}

The last concept that will be briefly discussed is gamma-gamma absorption. TeV gammarays that interact with optical photons exceed the threshold for electron-positron pair production, which will result in an absorption of the VHE gamma-rays. This idea has been used to explain the differences between the $\mathrm{GeV}$ and $\mathrm{TeV}$ light curves in some binaries, e.g. [36, 4] with detailed analysis undertaken by Dubus [60].

For PSR B1259-63, gamma-gamma absorption can provide an explanation for the dip in the $\mathrm{TeV}$ light curve around periastron. While the highly eccentric orbit of the binary supports the 

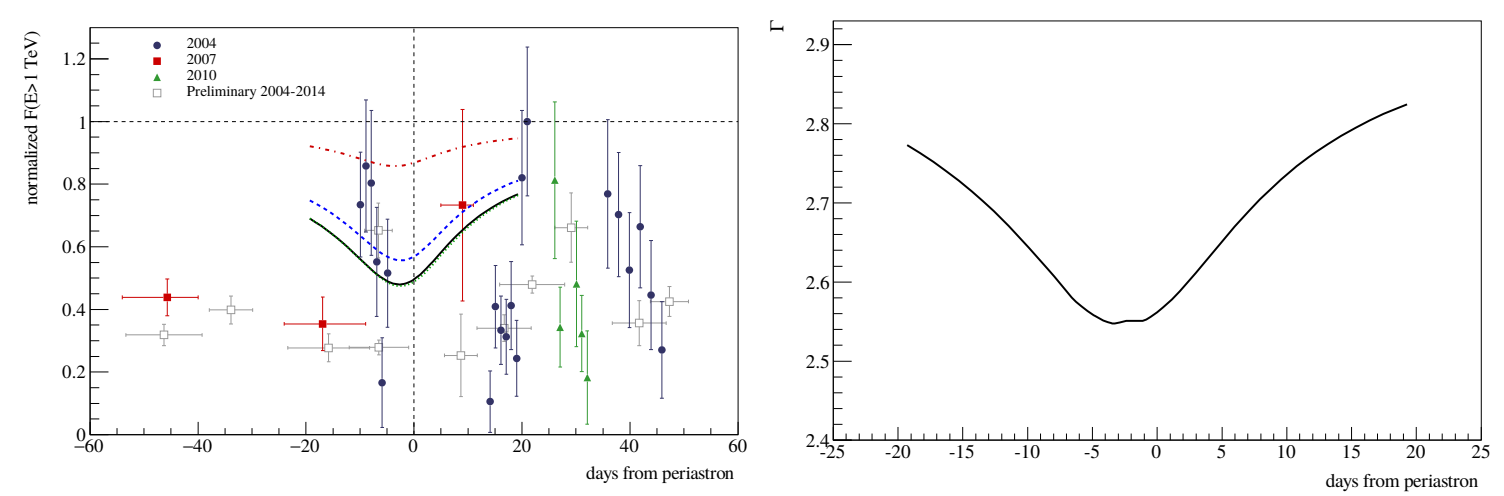

Figure 2: Left: The variation in the gamma-ray flux $(>1 \mathrm{TeV})$ around periastron passage in PSR B1259-63 assuming a constant intrinsic flux (photon index $\Gamma=3.0$ ). The gamma-gamma absorption due to the stellar (blue dashed line), disc (red dot-dashed line), and stellar+disc (solid black line) photons are shown. The results are compared to H.E.S.S. observations. Right: The induced variation in the gamma-ray photon index (1-50 TeV) for an intrinsic photon index of $\Gamma=3.0$. [61] (c) 2017. The American Astronomical Society. All rights reserved.)

emission increasing towards periastron, close to the actual periastron passage, gamma-gamma absorption could result in a large optical depth. A recent analysis of the the gamma-gamma absorption showed that the gamma-ray flux ( $>1 \mathrm{TeV}$ ) should, at $\approx 2$ days before periastron, decrease by $\approx 52 \%$ if both the stellar and circumstellar disc photons are taken into account [61]. The absorption due to the infrared photons from the disc alone results in $\approx 14 \%$ decrease in the flux. This is due to the fact that the peak in the gamma-gamma cross-section will occur at infrared frequencies ( $v \sim 10^{14} \mathrm{~Hz}$ for a $1 \mathrm{TeV}$ gamma ray) where the circumstellar disc provides additional photons. The gamma-gamma absorption would also introduce a hardening of the spectrum towards periastron (Fig. 2).

The gamma-gamma absorption may be an important tool for constraining the location of the gamma-ray production in these system. Independently produced relativistic hydrodynamic models of the wind interaction in gamma-ray binaries have shown that a "back shock" forms in the outflow moving away from the optical companion (Fig. 3; [62, 63, 64]). A second shock could provide a second location for particle acceleration and could explain some of the differences between the $\mathrm{GeV}$ and $\mathrm{TeV}$ emission. The observed $\mathrm{GeV}$ emission originates at the apex of the shock, while the observed $\mathrm{TeV}$ emission orginates from the "back shock" since the gamma-gamma absorption is too high near the apex $[65,66,33]$.

\section{Prospectives with CTA}

The CTA is expected to provide an order of magnitude improvement in sensitivity over current imaging air Cherenkov telescopes (IACTs). This improved sensitivity will be important for investigating such events as the orbit-to-orbit variation in the VHE light curves observed from LS I $+61^{\circ}$ 303, or improving the light curve of LMC P3 which has so far only been detected in one phase bin [4]. 


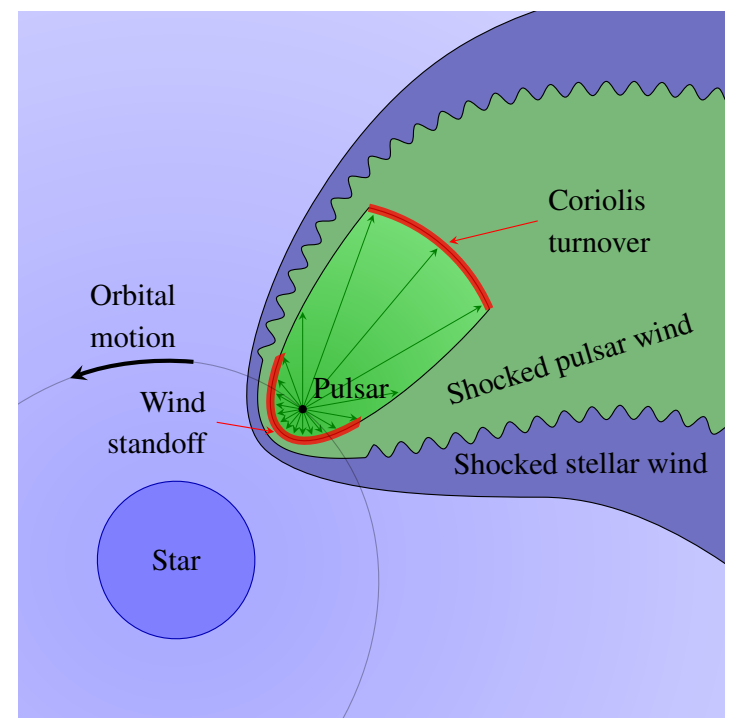

Figure 3: Sketch of the proposed orientation of the two shocks presented in Zabalza et al. [65]. The 'wind standoff' and the 'Coriolis turnover' are the two regions considered for particle acceleration. (Zabalza et al. A\&A, 551, A17, 2013, reproduced with permission (C) ESO.)

CTA could help constrain the location of the gamma-ray emission, since the improved statistics will allow us to better measure the short-term variation in the flux and photon index, and if this is caused by gamma-gamma absorption, this will place limits on the emission location.

The improved sensitivity will also be vital for discovering new sources. Two of the key science programmes for the CTA are the Galactic plane and LMC surveys [67]. These are key areas to detect new gamma-ray binaries. However, it should be cautioned that of the three most recently discovered gamma-ray binaries, one was a serendipitous H.E.S.S. discovery, while the other two where found by periodicity searches using Fermi-LAT. Once Fermi-LAT reaches the end of its mission (the mission is currently funded to at least the beginning of 2019), there will be no all-sky gamma-ray telescope operating in survey mode. A detailed population synthesis model suggests there are only $101_{-52}^{+89}$ gamma-ray binaries in the Galaxy and suggests only a few more sources will be discovered in the CTA era [68].

\section{Conclusions}

Gamma-ray binaries represent a unique opportunity to study the physical process of pulsar winds, shock fronts, particle acceleration etc. While the general broad-band emission can be explained by a pulsar producing a "nebula" within the binary system, the $\mathrm{GeV}$ component is more challenging and different interpretations have been put forward. The most complicated system to explain remains LS I $+61^{\circ} 303$ where studies have pointed to a jet structure.

There are still open questions regarding what influence the Be stars' circumstellar discs will have: the "infrared excess" introduces additional gamma-gamma absorption; in PSR B1259-63 the maxima in the X-ray and $\mathrm{TeV}$ light curves occur around the time the pulsar crosses the disc; there is a significant change in the disc size around the Fermi flare; in LS I $+61^{\circ} 303$ the disc varies with 
the super-orbital period. The up-coming CTA will help to resolve many of these outstanding issues, improving our understanding of the known systems, and discover new sources.

\section{References}

[1] VERITAS and MAGIC Collaborations 2017 TeV gamma-ray emission from PSR J2032+4127/ MT91 213 ATel 10810

[2] MAGIC and VERITAS Collaborations 2017 TeV gamma-ray emission from PSR J2032+4127/ MT91 213 at periastron ATel 10971

[3] R.H.D. Corbet et al. A luminous gamma-ray binary in the Large Magellanic Cloud, ApJ 829 (2016) 105

[4] H.E.S.S. collaboration Detection of variable VHE gamma-ray emission from the extra-galactic gamma-ray binary LMC P3 arXiv:1801.06322

[5] R.M. Shannon et al. The kinematics and orbital dynamics of the PSR B1259-63/LS 2883 system from 23 yr of pulsar timing, MNRAS 437 (2014) 3255

[6] I. Negueruela et al. Astrophysical parameters of LS 2883 and implications for the PSR B1259-63 gamma-ray binary, ApJL, 732 (2011) L11

[7] J. Casares et al. A possible black hole in the gamma-ray microquasar LS 5039, MNRAS 364 (2005) 899

[8] P.C. Gregory Bayesian analysis of radio observations of the Be X-ray binary LS I +61 ${ }^{\circ} 303$, ApJ 575 (2002) 427

[9] E.D. Grundstrom et al. Joint $H \alpha$ and $X$-ray observations of massive $X$-ray binaries. II The Be X-ray binary and microquasar $L S+61^{\circ} 303$, ApJ $\mathbf{6 5 6}$ (2007) 437

[10] C. Aragona et al. The orbits of the $\gamma$-ray binaries LS I +61 303 and LS 5039, ApJ 698 (2009) 514

[11] J. Casares et al. Orbital Parameters of the Microquasar LS I +61 303, MNRAS 360 (2005) 1105

[12] E. Aliu et al. Long-term TeV and X-ray observations of the gamma-ray binary HESS J0632+057, ApJ 780 (2014) 168

[13] J. Casares et al. On the binary nature of the $\gamma$-ray sources AGL J2241+4454 (= MWC 656) and HESS J0632+057 (= MWC 148), MNRAS 421 (2012) 1103

[14] H. An et al. Broadband X-ray properties of the gamma-ray binary 1FGL J1018.6-5856, ApJ 806 (2015) 166

[15] I. Monageng et al. The orbit of the gamma-ray binary 1FGL J1018.6-5856, ApJ 847 (2017) 68

[16] Fermi LAT collaboration Periodic Emission from the Gamma-ray Binary 1FGL J1018.6-5856, Science 335 (2012) 189

[17] F.D. Seward et al. DEM L241, A supernova remnant containing a high-mass X-ray binary, ApJ 759 (2012) 123

[18] W.C.G. Ho et al. Multiwavelength monitoring and X-ray brightening of Be X-ray binary PSR J2032+4127/MT91 213 on its approach to periastron, MNRAS 464 (2017) 1211

[19] A.G. Lyne et al. The binary nature of PSR J2032+4127, MNRAS 451 (2015) 581 
[20] Fermi LAT collaboration Modulated High-Energy Gamma-Ray Emission from the Microquasar Cygnus X-3, Science 326 (2009) 1512

[21] J. Albert et al. Very High Energy Gamma-Ray Radiation from the Stellar Mass Black Hole Binary Cygnus X-1, ApJ 665 (2007) 51

[22] M. Tavani et al. Regimes of high-energy shock emission from the Be star/pulsar system PSR 1259-63, ApJ 433 (1994) L37

[23] B.M. Gaensler \& P.O. Slane The Evolution and Structure of Pulsar Wind Nebulae, ARA\&A 44 (2006) 17

[24] G. Dubus Gamma-ray binaries: pulsars in disguise?, A\&A 456 (2006) 801

[25] S. Johnston et al. Radio observations of PSR B1259-64 around periastron, MNRAS 279 (1996) 1026

[26] V. Dhawan et al. $L S I+61^{\circ} 303$ is a Be-Pulsar binary, not a Microquasar in proceedings of The VI Microquasar Workshop: Microquasars and Beyond PoS(MQW6)052 (2006)

[27] M. Massi et al. VLBA images of the precessing jet of $L S I+61^{\circ} 303, A \& A \mathbf{5 4 0}$ (2012) A142

[28] Y.W. Wu et al. Revisiting LS I +61 303 with VLBI astrometry, MNRAS 474 (2018) 4245

[29] M. Massi \& G. Torricelli-Ciamponi Intrinsic physical properties and Doppler boosting effects in LS I $+61^{\circ} 303, A \& A 564$ (2014) A23

[30] S. Archambault et al. Exceptionally Bright TeV Flares from the Binary LS I +61 303, ApJ 817 (2016) L7.

[31] M. Actis et al. Design concepts for the Cherenkov Telescope Array CTA: an advanced facility for ground-based high-energy gamma-ray astronomy, Exp Astron 32 (2011) 193

[32] H. An \& R.W. Romani Light Curve and SED Modeling of the Gamma-Ray Binary 1FGL J1018.6-5856: Constraints on the Orbital Geometry and Relativistic Flow, ApJ 838 (2017) 145

[33] A.M. Chen et al. High-energy emissions from the gamma-ray binary 1FGL J1018.6-5856 (2017) [arXiv:1703.08080]

[34] P. Eger et al. Discovery of a variable X-ray counterpart to HESS J1832-093: a new gamma-ray binary?, MNRAS 457 (2016) 1753

[35] K. Mori NuSTAR Hard X-Ray Observation of the Gamma-Ray Binary Candidate HESS J1832-093, ApJ 848 (2016) 80

[36] A.A. Abdo, et al. Fermi/LAT observations of LS 5039, ApJ 706 (2009) L56

[37] F. Aharonian et al. 3.9 day orbital modulation in the TeV $\gamma$-ray flux and spectrum from the X-ray binary LS 5039, A\&A 460 (2006) 743

[38] A.A. Abdo et al. Discovery of high-energy gamma-ray emission from the binary system PSR B1259ấL Ś63/LS 2883 around periastron with Fermi, ApJL 736 (2011) L11

[39] G.A. Caliandro et al. Gamma-Ray Flare Activity from PSR B1259-63 during 2014 Periastron Passage and Comparison to Its 2010 Passage, ApJ 811 (2015) 68

[40] G. Dubus Gamma-ray binaries and related systems, A\&A Review 21 (2013) 64

[41] D. Khangulyan et al. Post-periastron Gamma-Ray Flare from PSR B1259-63/LS 2883 as a Result of Comptonization of the Cold Pulsar Wind, ApJ 752 (2012) L17

[42] G. Dubus \& B. Cerutti What caused the GeV flare of PSR B1259-63?, A\&A 557 (2013) A127. 
[43] M. Chernyakova et al. Multi-wavelength observations of the binary system PSR B1259-63/LS 2883 around the 2014 periastron passage, MNRAS 454 (2015) 1358

[44] S.V. Bogovalov et. al Modelling interaction of relativistic and non-relativistic winds in binary system PSR B1259-63/SS2883 - I. Hydrodynamical limit, MNRAS 387 (2008) 63

[45] G. Dubus et al. Relativistic Doppler-boosted emission in gamma-ray binaries, A\&A 516 (2010) A18

[46] S.W. Kong et al. Modeling the Multiwavelength Light Curves of PSR B1259-63/LS 2883. II. The Effects of Anisotropic Pulsar Wind and Doppler Boosting, ApJ 753 (2012) 127

[47] T. Rivinius et al. Classical Be stars. Rapidly rotating B stars with viscous Keplerian decretion disks, Astronomy and Astrophysics Review 21 (2013) 69

[48] S. Johnston et al. The 1997 periastron passage of the binary pulsar PSR B1259-63, MNRAS 326 (2001) 643

[49] S. Johnston et al. Radio observations of PSR B1259-63 through the 2004 periastron passage, MNRAS 358 (2005) 1069

[50] M. Chernyakova et al. Multiwavelength observations of the binary system PSR B1259-63/LS 2883 around the 2010-2011 periastron passage, MNRAS 439 (2014) 432

[51] B. van Soelen et al. Optical spectroscopy of PSR B1259-63/LS 2883 during the 2014 periastron passage with the Southern African Large Telescope, MNRAS 455 (2016) 3674

[52] R. Zamanov et al. $H \alpha$ observations of the $\gamma$-ray-emitting Be/X-ray binary $L S I+61^{\circ}$ 303:orbital modulation, disk truncation, and long-term variability, A\&A 559 (2013) A87

[53] B. van Soelen \& P.J. Meintjes The effect of the infrared excess from the Be star on inverse Compton gamma-ray production in PSR B1259-63/SS 2883, MNRAS 412 (2011) 1721

[54] B. van Soelen et al. Anisotropic inverse Compton scattering of photons from the circumstellar disc in PSR B1259-63, MNRAS 426 (2012) 3135

[55] A.T. Okazaki et al. Hydrodynamic Interaction between the Be Star and the Pulsar in the TeV Binary PSR B1259-63/LS 2883, PASJ 63 (2011) 893

[56] S.W. Kong et al. Modelling the multiwavelength light curves of PSR B1259-63/SS 2883, MNRAS 416 (2011) 1067

[57] J. Takata et al. Modeling High-energy Light curves of the PSR B1259-63/LS 2883 Binary Based on 3D SPH Simulations, ApJ 750 (2012) 70

[58] S.-X. Yi \& K.S. Cheng, Modelling the GeV emission of HESS J0632+057, MNRAS 471 (2017) 4228

[59] S.-X. Yi \& K.S. Cheng, A New Approach to the GeV Flare of PSR B1259-63/LS2883, ApJ 844 (2017) 114

[60] G. Dubus Gamma-ray absorption in massive X-ray binaries, A\&A 451 (2006) 9

[61] I. Sushch \& B. van Soelen Gamma-Gamma Absorption in the $\gamma$-ray Binary System PSR B1259-63/LS 2883, ApJ 837 (2017) 175

[62] V. Bosch-Ramon et al. Simulations of stellar/pulsar-wind interaction along one full orbit, A\&A 544 (2012) 59

[63] V. Bosch-Ramon et al. Orbital evolution of colliding star and pulsar winds in 2D and 3D: effects of dimensionality, EoS, resolution, and grid size, A\&A 577 (2015) A89 
[64] G. Dubus et al. Modelling the high-energy emission from gamma-ray binaries using numerical relativistic hydrodynamics, $A \& A \mathbf{5 8 1}$ (2015) A27

[65] V. Zabalza et al. Unraveling the high-energy emission components of gamma-ray binaries, A\&A $\mathbf{5 5 1}$ (2013) A17

[66] J. Takata et al. High-energy Emissions from the Gamma-Ray Binary LS 5039, ApJ 790 (2014) 18

[67] The Cherenkov Telescope Array Consortium Science with the Cherenkov Telescope Array arXiv: 1709.07997

[68] G. Dubus et al. Sizing up the population of gamma-ray binaries, A\&A 608 (2017) A59 